\title{
ESTADO NUTRICIONAL E QUALIDADE DE VIDA DE PACIENTES CANDIDATOS A TRANSPLANTE CARDÍACO
}

\author{
Nutritional status and quality of life of candidates for heart transplantation \\ Estado nutricional y calidad de vida de pacientes candidatos al trasplante cardíaco
}

\author{
Luana Najara Ferreira Chaves \\ Universidade de Fortaleza - UNIFOR - Fortaleza (CE) - Brasil \\ Hospital de Messejana Dr. Carlos Alberto Studart Gomes - Fortaleza (CE) - Brasil \\ Daniele Maria de Oliveira Carlos \\ Universidade de Fortaleza - UNIFOR - Fortaleza (CE) - Brasil \\ Hospital de Messejana Dr. Carlos Alberto Studart Gomes - Fortaleza (CE) - Brasil
}

\section{RESUMO}

Objetivo: Avaliar o estado nutricional e a qualidade de vida de pacientes candidatos a transplante cardíaco. Métodos: Estudo quantitativo e descritivo, com pacientes candidatos a transplante cardíaco atendidos no ambulatório de uma Unidade de Transplante e Insuficiência Cardíaca (UTIC) em Fortaleza, Ceará, Brasil. Coletou-se, através da entrevista, do atendimento e do prontuário, dados de peso, estatura, índice de massa corporal (IMC), circunferência braquial (CB), circunferência muscular do braço (CMB), dobra cutânea tricipital (DCT) e dados clínicos sobre a patologia de base. Aplicaram-se questionários de perfil socioeconômico, estilo de vida, frequência alimentar e qualidade de vida versão World Health Organization Quality of Life - WHOQOLBref. Utilizou-se estatística descritiva e frequências absoluta e relativa. As variáveis contínuas foram testadas através do coeficiente de correlação de Pearson. Resultados: A idade média foi de 50,64 12,45 anos e $57 \%(n=27)$ eram do sexo masculino. Na avaliação do estado nutricional (EN), 47,22\% ( $n=17)$ dos adultos tinham sobrepeso e 54,55\% ( $=6$ ) dos idosos eram eutróficos. Além disso, os adultos apresentaram-se eutróficos para CB, CMB e DCT, com 52,78\% ( $\mathrm{n}=19), 88,82 \%(\mathrm{n}=32)$ e $36,11 \%$ ( $n=13$ ), respectivamente. Os idosos também estavam eutróficos para CB e CMB, ambos com $81,82 \%$ ( $n=9$ ). A qualidade de vida (QV) mostrou-se satisfatória (regular) de acordo com a média dos domínios. Conclusão: Os pacientes apresentaram perfil nutricional de normalidade e/ou sobrepeso e uma qualidade de vida de regular a boa. As variações do EN e QV dependem da fase de vida e do apoio social/ familiar que recebem, atuando como determinantes positivos, mesmo na presença da doença cardíaca.

Descritores: Insuficiência Cardíaca; Estado Nutricional; Qualidade de Vida; Transplante de Coração.

\section{ABSTRACT}

Objective: To assess the nutritional status and quality of life of candidates for heart transplantation. Methods: Quantitative descriptive study of candiates for heart transplantation attending the outpatient clinic of a Heart Failure and Transplant Center (HFTC) in Fortaleza, Ceará, Brazil. Interviews, consultations and medical records were used to collect data on weight, height, Body mass index (BMI), arm circumference (AC), arm muscle circumference (AMC), triceps skinfold (TS) and clinical data on the underlying disease. Questionnaires addressing socioeconomic data, lifestyle and dietary frequency and the World Health Organization Quality of Life-WHOQOLBref were used. Descriptive statistics and absolute and relative frequencies were used. Continuous variables were tested using Pearson's correlation coefficient. Results: Participants' mean age was 50.64 \pm 12.45 years and $57 \%(n=27)$ were men. As for nutritional status, $47.22 \%(n=17)$ of the adults were overweight and $54.55 \%(n=6)$ of the older people were at normal weight. In addition, adults presented normal AC, AMC and TS, with $52.78 \%(n=19), 88.82 \%(n=32)$ and $36.11 \%(n=13)$, respectively. The older people also presented normal AC and AMC, both with $81.82 \%(n=9)$. Quality of life (QoL) was satisfactory (fair) according to the mean of the domains. Conclusion: The patients presented normal nutritional status and/or overweight and a quality of life ranging from fair to good. The variations in the NS and QoL depend on the life stage and on the social/family support they receive, which constitute positive determinants even in the presence of heart disease.

Descriptors: Heart Failure; Nutritional Status; Quality of Life; Heart Transplantation. 


\section{RESUMEN}

Objetivo: Evaluar el estado nutricional y la calidad de vida de pacientes candidatos al trasplante cardíaco. Métodos: Estudio cuantitativo y descriptivo con pacientes candidatos al trasplante cardiaco asistidos en el ambulatorio de una Unidad de Trasplante e Insuficiencia Cardiaca (UTIC) de Fortaleza, Ceará, Brasil. A través de la entrevista durante la asistencia y del historial clínico se recogieron los datos del peso, la estatura, el índice de masa corporal (IMC), la circunferencia braquial (CB), la circunferencia del músculo del brazo (CMB), el pliegue cutáneo tricipital (PCT) y los datos clínicos de la enfermedad de base. Se aplicaron cuestionarios sobre el perfil socioeconómico, el estilo de vida, la frecuencia alimentaria y la calidad de vida de la versión World Health Organization Quality of Life - WHOQOLBref. Se utilizó la estadística descriptiva y las frecuencias absoluta y relativa. Las variables continuas fueron testadas a través del coeficiente de correlación de Pearson. Resultados: La media de la edad fue de 50,64 12,45 años y el 57\% (n=27) eran del sexo masculino. En la evaluación del estado nutricional (EN), el 47,22\% (n=17) de los adultos tenían sobrepeso y el 54,55\% (n=6) de los mayores eran eutróficos. Además de eso, los adultos se presentaron eutróficos para la CB, la CMB y el PCT con el $52,78 \%(n=19)$, el $88,82 \%(n=32)$ y el $36,11 \%(n=13)$ respectivamente. Los mayores también estaban eutróficos para la CB y la CMB, ambos con el 81,82\% (n=9). La calidad de vida (CV) se mostró satisfactoria (regular) según la media de los dominios. Conclusión: Los pacientes presentaron el perfil nutricional de normalidad y/o sobrepeso y una calidad de vida de regular a buena. Las variaciones del EN y de la CV dependen de la fase de la vida y del apoyo socialffamiliar que reciben actuando como determinantes positivos incluso en la presencia de enfermedad cardiaca.

Descriptores: Insuficiencia Cardíaca; Estado Nutricional; Calidad de Vida; Trasplante de Corazón.

\section{INTRODUÇÃO}

O transplante cardíaco (TC) é o tratamento de escolha para a insuficiência cardíaca (IC) refratária, apesar da melhora da expectativa de vida desses pacientes e do tratamento clínico realizado nas últimas décadas. Vários foram os avanços nessa área, incluindo a incorporação de novas técnicas cirúrgicas, novos imunossupressores, novos métodos diagnósticos e abordagens nos pós-operatórios precoce e tardio ${ }^{(1,2)}$.

A IC é definida como disfunção cardíaca que ocasiona inadequado suprimento sanguíneo para atender às necessidades metabólicas tissulares na presença de retorno venoso normal, ou com elevadas pressões de enchimento ${ }^{(2)}$. Ela é um importante problema de saúde pública e vem sendo considerada como uma nova epidemia de elevada morbimortalidade. No Brasil, observase um aumento das hospitalizações por doenças cardíacas e a IC é a principal causa delas, pois configura a principal causa de internação na população idosa maior de 65 anos, correspondendo a $80 \%$ das hospitalizações. Devido ao envelhecimento da população brasileira, observa-se um potencial crescimento de pacientes em risco ou portadores de $\mathrm{IC}^{(2,3)}$.

A evolução clínica dos pacientes com IC, normalmente, leva a uma série de alterações fisiológicas, das quais muitas influenciam diretamente o estado nutricional ${ }^{(4)}$, que, além de ser considerado um dos múltiplos fatores envolvidos na etiologia da doença, está relacionado à progressão e piores desfechos clínicos em portadores de $\mathrm{IC}^{(5)}$.

O catabolismo e hipermetabolismo se agravam com a progressão da doença, podendo levar ao quadro de caquexia cardíaca. As alterações no estado nutricional também podem ser ocasionadas pela menor ingestão alimentar e aproveitamento de nutrientes ${ }^{(5,6)}$. Dentre as alterações que ocorrem nessa condição e influenciam na ingestão e aceitação alimentar estão: as do trato gastrointestinal, como compressão gástrica e congestão hepática, que geram sensação de plenitude pós-prandial; edema de alças intestinais, que diminui a capacidade absortiva, principalmente das proteínas (situação conhecida como enteropatia perdedora de proteína); além de anorexia, náuseas, dispneia e fadiga ${ }^{(6)}$.

Diante disso, os pacientes apresentam frequentemente perda ponderal, associada a uma diminuição da reserva energética e perda da massa muscular progressiva, podendo a perda de peso chegar até $20 \%$ de forma involuntária e $6 \%$ de massa muscular em seis meses. Essa perda muscular traz prejuízos à mobilidade e capacidade funcional $(\mathrm{CF})$, além de piorar o prognóstico, aumentar as complicações pós-operatórias e a mortalidade ${ }^{(4,6)}$.

Pode-se dizer que a IC assume um grande impacto nas diferentes esferas da vida dos pacientes, com o comprometimento emocional, social e econômico, além de limitação física. Dessa forma, a qualidade de vida (QV) estará prejudicada e o contexto geral da vida diária será afetado pela incapacidade física que a doença cardíaca traz ${ }^{7,8)}$.

Com base no exposto, o objetivo do presente estudo foi avaliar o estado nutricional e a qualidade de vida de pacientes candidatos a transplante cardíaco.

\section{MÉTODOS}

Trata-se de uma pesquisa de natureza quantitativa e descritiva, com pacientes candidatos a transplante cardíaco atendidos no ambulatório da Unidade de Transplante e Insuficiência Cardíaca (UTIC) de um hospital referência no tratamento de doenças cardíacas na cidade de Fortaleza, Ceará, Brasil, realizada em 2016. 
O universo da pesquisa partiu do levantamento dos atendimentos realizados no ano de 2015, totalizando em média 120 pacientes atendidos. A amostra foi composta pelos pacientes que compareceram aos atendimentos no período de coleta (julho a dezembro de 2016) e atenderam aos critérios de inclusão, totalizando no estudo 47 pacientes.

Incluíram-se os pacientes com idade maior ou igual a 20 anos, de ambos os sexos, acompanhados no ambulatório da UTIC e que aceitaram participar da pesquisa mediante a assinatura do Termo de Consentimento Livre e Esclarecido (TCLE). Excluíram-se os pacientes com alteração do grau de consciência e/ou incapazes de responder aos dados da pesquisa.

A coleta dos dados ocorreu pela avaliação nutricional, dados clínicos sobre a patologia de base e aplicação de três questionários: socioeconômico e estilo de vida, frequência alimentar e outro sobre qualidade de vida (World Health Organization Quality of Life -WHOQOLBref).

Para avaliação do estado nutricional, realizaram-se aferições de peso, estatura, circunferência braquial (CB), circunferência muscular do braço (CMB), dobra cutânea tricipital (DCT) e cálculo do Índice de Massa Corporal (IMC). Para aferição do peso, o paciente subiu no centro da balança digital (Tanita ${ }^{\circledR}$ ), em pé, descalço e com roupas leves. A aferição da estatura ocorreu com o indivíduo em pé, ereto, descalço, com os calcanhares juntos, costas eretas e braços estendidos ao lado do corpo, através de estadiômetro (Standart Sanny $\left.{ }^{\circledR}\right)^{(6)}$.

A partir dos dados coletados de peso e estatura, calculou-se o IMC (peso/estatura ${ }^{2}$ ). Para interpretação desse dado nos pacientes dentro da faixa etária adulta, considerou-se os padrões de referência da Organização Mundial de Saúde, que atendem aos limites: $<18,5 \mathrm{~kg} / \mathrm{m}^{2}$ para baixo peso, 18,5 a $24,9 \mathrm{~kg} / \mathrm{m}^{2}$ para eutrofia e $>24,9 \mathrm{~kg} / \mathrm{m}^{2}$ para sobrepeso, ou obesidade, para indivíduos adultos ${ }^{(9)}$. Para avaliação do IMC de idosos, utilizou-se os limites: $<22 \mathrm{~kg} / \mathrm{m}^{2}$ para baixo peso, 22 a $27 \mathrm{~kg} / \mathrm{m}^{2}$ eutrofia e $>27 \mathrm{~kg} / \mathrm{m}^{2}$ para excesso de peso $^{(10)}$.

Para a mensuração da circunferência braquial (CB), utilizou-se uma fita métrica inelástica na linha média entre o acrômio da escápula e o olécrano da ulna, em estado de relaxamento ao longo do corpo. A mensuração da dobra cutânea tricipital (DCT) foi obtida com adipômetro (Standart Sanny ${ }^{\circledR}$ ) e a leitura feita no milímetro mais próximo ${ }^{(6)}$. A partir da aferição da CB e da DCT, obteve-se a medida da circunferência muscular do braço $(\mathrm{CMB})$, a qual foi calculada através da fórmula $\mathrm{CMB}(\mathrm{cm})=\mathrm{CB}$ (cm) - [0,314 x DCT (mm)]. A interpretação dos resultados de CB, CMB e DCT foi realizada através do cálculo de percentual de adequação das variáveis ${ }^{(11)}$.

Para avaliação do perfil clínico, aplicou-se o protocolo de avaliação de cadastro de candidato a transplante cardíaco, utilizado no ambulatório da UTIC e adaptado para a atual pesquisa; além de consulta aos prontuários dos pacientes, obtendo-se, assim, as informações sobre a patologia, a etiologia, as comorbidades associadas e a classificação funcional da IC.

Para traçar o perfil demográfico dos pacientes, aplicou-se o questionário socioeconômico e estilo de vida. Trata-se de uma ficha de cadastro já utilizada na UTIC, porém adaptada com perguntas referentes ao estilo de vida, questionando-se informações sobre sexo, idade, procedência, religião, ocupação, renda, escolaridade e estilo de vida, como a prática de exercício físico, tabagismo e etilismo. Algumas dessas informações também foram consultadas nos prontuários.

Na avaliação dos hábitos alimentares, realizou-se uma entrevista com os pacientes na qual foi aplicado o questionário de frequência alimentar (QFA), obtendo-se informações qualitativas sobre o padrão de consumo alimentar ${ }^{(12)}$.

A avaliação dos hábitos alimentares seguiu os parâmetros da Pirâmide Alimentar Brasileira, considerando-se adequadas as porções com as seguintes quantidades: três ou mais porções/dia de frutas; três ou mais porções/dia de verduras e legumes; uma porção/dia de bfeijões e oleaginosas; uma porção/dia de carnes e ovos; seis porções/dia de arroz, pão, massa, batata e mandioca; três porções/dia de leite, queijo e iogurte. A classificação do consumo alimentar foi definida como adequada (consumo diário) e inadequada (semanal/raramente/nunca). Essas informações foram coletadas por meio de entrevista com os pacientes ${ }^{(13)}$.

Para avaliação da qualidade de vida, utilizou-se o questionário estruturado e padronizado, o World Health Organization Quality of Life (WHOQOLBref), na versão abreviada e em português ${ }^{(14,15)}$, que avalia também a percepção dos indivíduos a respeito do impacto que as doenças causam em suas vidas. O WHOQOL-Bref em sua versão final ficou composto por 26 questões fechadas. A primeira questão refere-se à qualidade de vida de modo geral, a segunda, à satisfação com a própria saúde e as outras 24 são representantes de cada uma das 24 facetas que compõem o instrumento original. Essas 24 questões são divididas em 4 domínios: físico (7 itens), psicológico (6 itens), relações sociais ( 3 itens) e meio ambiente ( 8 itens) ${ }^{(14)}$.

Cada domínio foi categorizado em três níveis de satisfação (baixo, médio e alto) para fins de análise ${ }^{(16)}$. Essa escala é categorizada da seguinte maneira: valores entre 0 e 40 são considerados região de insatisfação, ou que precisa melhorar; de 41 a 69, correspondem à região de indefinição, ou regular; e, acima de 70, como tendo atingido a região de sucesso, ou seja, muito boa qualidade de vida ${ }^{(17,18)}$.

Todos os dados obtidos foram expressos por meio de estatísticas descritivas (média e desvio padrão) e frequências absoluta e relativa. No tratamento estatístico dos resultados, utilizou-se o coeficiente de correlação linear de Pearson, obtido através do software estatístico R-Studio ${ }^{\circledR}$. Os resultados foram apresentados por meio de tabelas e gráficos de acordo com as variáveis em estudo.

Esta pesquisa recebeu aprovação pelo Comitê de Ética em Pesquisa do hospital onde a pesquisa ocorreu, sob Parecer $\mathrm{n}^{\circ}$ 1.639.432, e baseou-se na Resolução 466/12 da Comissão Nacional de Ética em Pesquisa (Conep) do Conselho Nacional de Saúde (CNS), sendo resguardados o anonimato e a privacidade dos pesquisados. 


\section{RESULTADOS}

A idade da população em estudo apresentou média de 50,64 $\pm 12,45$ anos, no qual $75 \%(n=35)$ dos pacientes apresentou idade inferior a 59 anos, portanto adultos. Em relação à variável sexo, 57\% (n=27) eram do sexo masculino. Quando avaliados em relação à escolaridade, ocupação, renda e procedência, verificou-se que: $63,82 \%(n=30)$ dos pacientes tinham até o ensino fundamental; $59,57 \%(\mathrm{n}=28)$ não trabalhavam por motivos de limitação física associada à patologia de base, mas ainda não eram aposentados; e 55,32\% (n=26) tinham renda mensal de apenas 1 salário mínimo ou menos (Tabela I).

No que se refere à procedência, $55 \%(n=26)$ eram provenientes da capital, Fortaleza, e apenas $11 \%(n=5)$ eram procedentes de outros estados e compareciam à capital nos dias de atendimento, ou permaneciam por alguns meses para o tratamento e/ou estabilidade da doença (Tabela I).

Em relação aos hábitos associados ao estilo de vida, embora a maioria não fosse tabagista nem etilista, houve uma parcela importante de ex-tabagistas $(40,43 \%, \mathrm{n}=19)$ e ex-etilistas $(44,68 \%, \mathrm{n}=21)$, o que merece ser considerado. Dos pacientes avaliados, apenas $10,64 \%(\mathrm{n}=5)$ praticavam algum tipo de atividade física, o que pode ser justificado pela limitação física da doença de base (Tabela I).

No tocante ao diagnóstico clínico associado à IC, a prevalência de miocardiopatia idiopática e miocardiopatia isquêmica foram predominantes, cada uma com 38,3\% $(\mathrm{n}=18)$. Os principais fatores de risco cardiovascular associados ao diagnóstico clínico foram: a Hipertensão Arterial Sistêmica (HAS), presente em 57,45\% (n=27) dos pacientes; história familiar de doenças cardíacas, 48,97\% (n=23); e IAM (Infarto Agudo do Miocárdio) prévio, presente em 36,17\% (n=17) (Tabela I).

Considerando os fatores de descompensação da IC, o esforço físico $(64,91 \%, \mathrm{n}=31)$ e a HAS $(23,68 \%, \mathrm{n}=11)$ foram os principais. A maioria dos pacientes tinha em torno de 5 anos de doença $(40,43 \%, \mathrm{n}=19)$.

A avaliação da classificação funcional da IC mostrou que 59,57\% ( $\mathrm{n}=28)$ dos pacientes encontravam-se na classe II, mas 40,53\% ( $\mathrm{n}=19)$ encontravam-se nas classes III e IV, que têm indicação de transplante. Dos pacientes investigados, $72,97 \%$ $(\mathrm{n}=34)$ não tiveram internação nos últimos 12 meses, estando com a doença sob controle (Tabela I).

Tabela I - Perfil demográfico e clínico de pacientes candidatos a transplante cardíaco em um hospital de referência no tratamento de doenças cardíacas na cidade de Fortaleza, Ceará, 2016.

\begin{tabular}{lcc}
\hline Dados & Quantidade & Frequência \\
\hline Escolaridade & & \\
Analfabeto & 3 & $6,38 \%$ \\
Ensino fundamental & 27 & $57,44 \%$ \\
Ensino médio & 12 & $25,53 \%$ \\
Superior & 5 & $10,64 \%$ \\
Ocupação & & \\
Aposentado & 18 & $38,30 \%$ \\
Trabalha & 1 & $2,13 \%$ \\
Não trabalha & 28 & $59,57 \%$ \\
Renda & & \\
<1 salário & 26 & $55,32 \%$ \\
1 a 2 salários & 14 & $29,79 \%$ \\
3 a 4 salários & 4 & $8,51 \%$ \\
> 4 salários & 3 & $6,38 \%$ \\
Procedência & & \\
Capital: Fortaleza - Ceará & 26 & $55,00 \%$ \\
Municípios - Ceará & 16 & $34,00 \%$ \\
Outros estados & 5 & $11,00 \%$ \\
Hábitos relacionados ao estilo de vida & & \\
Não tabagista & 28 & $59,57 \%$ \\
Ex-tabagista & 19 & $40,43 \%$ \\
Não etilista & 26 & $55,32 \%$ \\
Ex-etilista & 21 & $44,68 \%$ \\
Diagnóstico clínico & & \\
Miocardiopatia dilatada idiopática & 18 & $38,30 \%$ \\
Miocardiopatia isquêmica & 18 & $38,30 \%$ \\
Miocardiopatia periparto & 5 & $10,64 \%$ \\
Miocardiopatia por doença valvar & 3 & $6,38 \%$ \\
Outras (chagásica, alcoólica e hipertensiva) & 3 & $6,36 \%$ \\
\hline
\end{tabular}




\begin{tabular}{lcc}
\hline Fatores de risco & & \\
HAS & 27 & $57,45 \%$ \\
Diabetes & 9 & $19,15 \%$ \\
IAM prévio & 17 & $36,17 \%$ \\
Insuficiência renal & 3 & $6,38 \%$ \\
História familiar & 23 & $48,97 \%$ \\
Revascularização miocárdica & 7 & $14,89 \%$ \\
Cirurgia cardíaca & 12 & $25,53 \%$ \\
Troca valvar & 1 & $2,13 \%$ \\
Dislipidemia & 11 & $23,40 \%$ \\
Menopausa c/reposição & 6 & $28,57 \%$ \\
Menopausa s/reposição & 10 & $50,0 \%$ \\
Atividade física & 5 & $10,64 \%$ \\
Fatores de descompensação & & \\
HAS & 11 & $23,68 \%$ \\
Isquemia & 2 & $3,30 \%$ \\
Arritmia & 3 & $6,38 \%$ \\
Insuficiência renal & 1 & $2,13 \%$ \\
Progressão da doença de base & 8 & $16,46 \%$ \\
Esforço físico & 31 & $64,91 \%$ \\
Tempo de IC & & \\
< 6 meses & 4 & $8,51 \%$ \\
1 a 2 anos & 18 & $32,29 \%$ \\
2 a 3 anos & 6 & $12,77 \%$ \\
5 anos & 19 & $40,43 \%$ \\
Classe funcional da IC & & \\
Classe I & 0 & $0 \% \%$ \\
Classe II & 28 & $59,57 \%$ \\
Classe III & 17 & $36,17 \%$ \\
Classe IV & 2 & $4,26 \%$ \\
Internação nos últimos 12 meses & & \\
Não & 34 & \\
Sim & 13 & \\
\hline & & \\
\hline & & \\
\hline
\end{tabular}

IC: Insuficiência cardíaca; HAS: Hipertensão arterial sistêmica; IAM: Infarto Agudo do Miocárdio

A análise do estado nutricional (EN) da amostra, considerando os critérios específicos para adultos e idosos, evidenciou que 74,99\% $(n=27)$ dos pacientes adultos encontravam-se com excesso de peso, com predominância para o sobrepeso 47,22\% $(\mathrm{n}=17)$. Já entre os idosos predominou a eutrofia $54,55 \%(\mathrm{n}=6)$, mas com uma parcela importante com excesso de peso $36,36 \%$ $(\mathrm{n}=4)$ (Tabela II).

Os resultados de adequação das variáveis $\mathrm{CB}, \mathrm{CMB}$, e DCT também foram expressos por classificação etária para adulto e idoso. No caso da CB, 52,78\% $(n=19)$ dos adultos e 81,82\% $(n=9)$ dos idosos apresentaram maior prevalência de eutrofia. Em relação à $\mathrm{CMB}$, foram $88,89 \%(\mathrm{n}=32)$ dos adultos e $81,82 \%(\mathrm{n}=9)$ dos idosos com eutrofia. Já para a DCT, embora não tenha predominado a eutrofia, houve uma parcela muito importante de eutrofia, de $36,11 \%(n=13)$ nos adultos e $36,36 \%$ ( idosos (Tabela II). 
Tabela II - Estado nutricional de pacientes candidatos a transplante cardíaco de acordo com as variáveis Índice de Massa Corporal (IMC), Circunferência do Braço (CB), Circunferência Muscular do Braço (CMB) e Dobra Cutânea Triciptal (DCT), segundo faixa etária. Fortaleza, Ceará, 2016.

\begin{tabular}{|c|c|c|}
\hline Estado Nutricional & Quantidade & Frequência \\
\hline \multicolumn{3}{|l|}{ IMC adulto } \\
\hline Desnutrição grave & 0 & $0,00 \%$ \\
\hline Desnutrição moderada & 1 & $2,78 \%$ \\
\hline Desnutrição leve & 1 & $2,78 \%$ \\
\hline Eutrofia & 7 & $19,44 \%$ \\
\hline Sobrepeso & 17 & $47,22 \%$ \\
\hline Obesidade leve & 3 & $8,33 \%$ \\
\hline Obesidade moderada & 3 & $8,33 \%$ \\
\hline Obesidade grave & 4 & $11,11 \%$ \\
\hline Total & 36 & $100 \%$ \\
\hline \multicolumn{3}{|l|}{ IMC idoso } \\
\hline Baixo peso & 1 & $9,09 \%$ \\
\hline Eutrofia & 6 & $54,55 \%$ \\
\hline Sobrepeso & 4 & $36,36 \%$ \\
\hline Total & 11 & $100 \%$ \\
\hline \multicolumn{3}{|c|}{ Adequação da CB (\%) - Adulto } \\
\hline Obesidade & 5 & $13,88 \%$ \\
\hline Sobrepeso & 3 & $8,33 \%$ \\
\hline Eutrofia & 19 & $52,78 \%$ \\
\hline Desnutrição leve & 5 & $13,88 \%$ \\
\hline Desnutrição moderada & 4 & $11,11 \%$ \\
\hline Desnutrição grave & 0 & $0,00 \%$ \\
\hline Total & 36 & $100 \%$ \\
\hline \multicolumn{3}{|c|}{ Adequaç̃o da CB (\%) - Idoso } \\
\hline Obesidade & 0 & $0,00 \%$ \\
\hline Sobrepeso & 1 & $9,09 \%$ \\
\hline Eutrofia & 9 & $81,82 \%$ \\
\hline Desnutrição leve & 0 & $0,00 \%$ \\
\hline Desnutrição moderada & 1 & $9,09 \%$ \\
\hline Desnutrição grave & 0 & $0,00 \%$ \\
\hline Total & 11 & $100 \%$ \\
\hline \multicolumn{3}{|c|}{ Adequação da CMB (\%) - Adulto } \\
\hline Eutrofia & 32 & $88,89 \%$ \\
\hline Desnutrição leve & 2 & $5,56 \%$ \\
\hline Desnutrição moderada & 2 & $5,56 \%$ \\
\hline Desnutrição grave & 0 & $0,00 \%$ \\
\hline Total & 36 & $100 \%$ \\
\hline \multicolumn{3}{|c|}{ Adequação da CMB (\%) - Idoso } \\
\hline Eutrofia & 9 & $81,82 \%$ \\
\hline Desnutrição leve & 1 & $9,09 \%$ \\
\hline Desnutrição moderada & 1 & $9,09 \%$ \\
\hline Desnutrição grave & 0 & $0,00 \%$ \\
\hline Total & 11 & $100 \%$ \\
\hline \multicolumn{3}{|c|}{ Adequação da DCT (\%) - Adulto } \\
\hline Obesidade & 8 & $22,22 \%$ \\
\hline Sobrepeso & 1 & $2,78 \%$ \\
\hline Eutrofia & 13 & $36,11 \%$ \\
\hline Desnutrição leve & 6 & $16,67 \%$ \\
\hline Desnutrição moderada & 1 & $2,78 \%$ \\
\hline Desnutrição grave & 7 & $19,44 \%$ \\
\hline Total & 36 & $100 \%$ \\
\hline \multicolumn{3}{|c|}{ Adequação da DCT (\%) - Idoso } \\
\hline Obesidade & 4 & $36,36 \%$ \\
\hline Sobrepeso & 0 & $0,00 \%$ \\
\hline Eutrofia & 4 & $36,36 \%$ \\
\hline Desnutrição leve & 0 & $0,00 \%$ \\
\hline Desnutrição moderada & 2 & $18,19 \%$ \\
\hline Desnutrição grave & 1 & $9,09 \%$ \\
\hline Total & 11 & $100 \%$ \\
\hline
\end{tabular}


A avaliação do padrão alimentar através da aplicação do QFA demonstrou que os grupos alimentares com adequado consumo, considerando a frequência diária, foram: grupo carnes e ovos $(66,12 \%, n=30)$ e grupo arroz, pão, massa, batata e mandioca $(65,72 \%, \mathrm{n}=31)$.

Já os principais grupos alimentares de consumo inadequado (semanal/raramente/nunca) foram: grupo leite, queijo e iogurte $(87,75 \%, n=41)$, grupo feijões e oleaginosas $(87,36 \%, n=41)$, grupo óleos e gorduras $(78,23 \%, n=37)$, grupo açúcares e doces $(81,97 \%, n=39)$, grupo frutas,verduras e legumes $(54,42 \%, n=26)$. Os resultados obtidos sobre a frequência de consumo dos grupos alimentares podem ser associados na avaliação dos desfechos clínicos da doença ou no surgimento de comorbidades relacionadas às doenças crônicas não transmissíveis.

A análise da qualidade de vida foi feita com base na média dos escores obtidos de cada domínio avaliado pelo questionário. O domínio físico apresentou menor pontuação $(54,56)$, evidenciando que esse é o domínio mais afetado devido à limitação física que a doença traz. As perguntas sobre a qualidade de vida, de um modo geral, foram incluídas em outro domínio para facilitar a compreensão dos resultados, sendo denominado domínio de autoavaliação da qualidade de vida. Esse apresentou a segunda menor pontuação em relação aos demais $(57,71)$ e reflete uma satisfação regular com a qualidade de vida, de um modo geral. Os domínios que apresentaram maiores médias foram: psicológico $(65,02)$ e relações sociais $(67,73)$. Fatores estes que impactam de forma importante na autoestima dos pacientes e, consequentemente, na qualidade de vida. Neste caso, o domínio psicológico e de relações sociais contribuíram positivamente para que os indivíduos considerassem sua vida de regular a boa, mesmo com o acometimento da doença cardíaca (Tabela III).

Tabela III - Análise descritiva dos domínios de qualidade de vida (QV) de pacientes candidatos a transplante cardíaco em um hospital de referência no tratamento de doenças cardíacas através da análise descritiva dos domínios. Fortaleza, Ceará, 2016.

\begin{tabular}{lccccccc}
\hline Domínios & Média & $\begin{array}{c}\text { Desvio } \\
\text { Padrão }\end{array}$ & $\begin{array}{c}\text { Coeficiente } \\
\text { de Variação }\end{array}$ & $\begin{array}{c}\text { Valor } \\
\text { Mínimo }\end{array}$ & $\begin{array}{c}\text { Valor } \\
\text { Máximo }\end{array}$ & $\begin{array}{c}\text { Amplitude } \\
\text { Escala } \\
\text { (0 a 100) }\end{array}$ \\
\hline Físico & 12,73 & 2,28 & 17,88 & 7,43 & 16,57 & 9,14 & 54,56 \\
Psicológico & 14,40 & 2,33 & 16,21 & 8,67 & 18,00 & 9,33 & 65,02 \\
Relações sociais & 14,84 & 2,25 & 15,18 & 6,67 & 17,33 & 10,67 & 67,73 \\
Meio ambiente & 13,84 & 2,09 & 15,09 & 8,50 & 17,50 & 9,00 & 61,52 \\
Autoavaliação da QV & 13,23 & 3,11 & 23,49 & 6,00 & 20,00 & 14,00 & 57,71 \\
Geral & 13,71 & 1,90 & 13,87 & 8,15 & 16,92 & 8,77 & 60,71 \\
\hline
\end{tabular}

A figura 1 apresenta os resultados dos escores médios obtidos por cada faceta de perguntas, possibilitando uma análise mais detalhada. Na análise das facetas, observou-se que 84,04\% (n=39) dos pacientes são dependentes do uso de medicações e/ou necessitam de acompanhante durante as consultas clínicas. Quanto à presença, ou não, de dores ou desconforto, 41,49\% $(n=20)$ dos pacientes sentiam algum tipo de dor ou desconforto, com destaque para o cansaço e falta de energia relatada pelos pacientes, o que interfere diretamente na execução das suas atividades básicas diárias. Em relação aos aspectos positivos, podese destacar: autoestima $(78,72 \%, \mathrm{n}=37)$, suporte (apoio) familiar $(72,34 \%, \mathrm{n}=34)$, bom convívio no lar (ambiente) $(71,28 \%$, $n=34)$, relações sociais e espiritualidade/religião/crenças pessoais, ambos com $69,68 \%$ ( $n=33)$.

As duas primeiras perguntas do questionário são referentes à qualidade de vida de um modo geral (considerando "muito ruim", "ruim", "nem muito ruim nem muito boa", "boa" e "muito boa") e a satisfação com a própria saúde (considerando "muito insatisfeito", "insatisfeito", "nem insatisfeito nem satisfeito", "satisfeito" e "muito satisfeito"). Os domínios físico e psicológico apresentaram valores invertidos de pontuação em algumas questões, cuja interpretação mostra que, quanto menor for a resposta, melhor avaliado será o paciente. Os resultados das respostas de cada faceta podem, portanto, ser aplicados em uma escala normal $\left(\begin{array}{lllll}1 & 2 & 3 & 4 & 5\end{array}\right)$ ou invertida $\left(\begin{array}{lllll}5 & 4 & 3 & 2 & 1\end{array}\right)^{(18)}$. Os escores obtidos demostraram regular satisfação com a saúde e qualidade de vida de um modo geral. A figura 1 apresenta essas facetas de pontuação invertida com a barra na cor preta. 


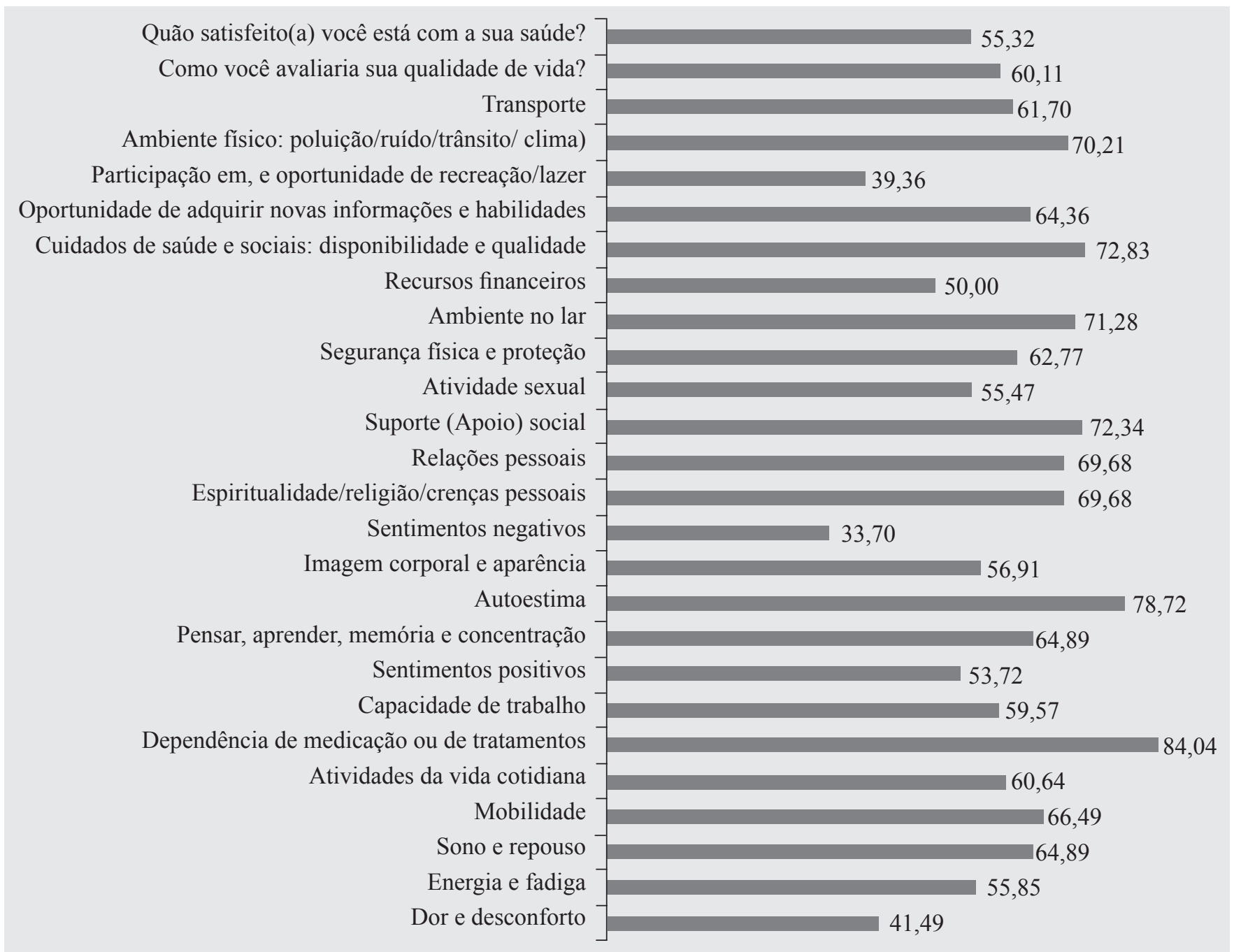

Figura1 - Qualidade de vida de pacientes candidatos a transplante cardíaco em um hospital de referência no tratamento de doenças cardíacas, segundo escore por facetas. Fortaleza, Ceará, 2016.

Para a análise de associação entre as variáveis quantitativas (medidas antropométricas x domínios), utilizou-se o coeficiente de Correlação Linear de Pearson que assume valores entre -1 e 1. Diante dos resultados apresentados na Tabela IV, verificou-se que existe uma correlação linear direta entre DCT e domínio psicológico, e inversamente proporcional entre estatura e domínio qualidade de vida, mas ela não se apresenta forte entre as variáveis testadas.

O coeficiente de correlação linear entre as variáveis qualidade de vida e medidas antropométricas mediu o grau da correlação (e a direção dessa relação - se positiva ou negativa). Os valores perto de -1 indicam que existe uma perfeita relação inversamente proporcional entre as variáveis, a proximidade da correlação a 1 indica que há uma perfeita relação linear diretamente proporcional entre as variáveis, e a proximidade da correlação de 0 mostra que não existe correlação linear entre as variáveis.

Tabela IV - Correlação entre as medidas antropométricas e os domínios associados à qualidade de vida de pacientes candidatos a transplante cardíaco. Fortaleza, Ceará, 2016.

\begin{tabular}{lccccc}
\hline \multirow{2}{*}{ Medidas Antropométricas } & \multicolumn{4}{c}{ Domínios (qualidade de vida) } \\
\cline { 2 - 6 } & Físico & Psicológico & $\begin{array}{c}\text { Relações } \\
\text { Sociais }\end{array}$ & $\begin{array}{c}\text { Meio } \\
\text { Ambiente }\end{array}$ & $\begin{array}{c}\text { Qualidade de } \\
\text { Vida }\end{array}$ \\
\hline Peso Aferido & 0,0199 & 0,0066 & 0,1155 & 0,0404 & 0,0483 \\
Altura & 0,1274 & 0,2235 & 0,2641 & 0,1465 & 0,3127 \\
Circunferência Braquial & $-0,0572$ & $-0,1293$ & 0,0641 & $-0,0369$ & $-0,0273$ \\
Circunferência Muscular do Braço & $-0,0362$ & $-0,0132$ & 0,0866 & 0,0065 & 0,0215 \\
Dobra Cutânea Tricipital & $-0,0317$ & $-0,2458$ & $-0,1446$ & $-0,0161$ & $-0,0203$ \\
\hline
\end{tabular}

* Para avaliação da correlação entre as variáveis foi utilizada a Correlação Linear de Pearson. 


\section{DISCUSSÃO}

A média de idade encontrada no presente estudo foi de 50,64 $\pm 12,45$ anos, com predominância de adultos de meia idade, o que pode ser justificado pelo envelhecimento da população brasileira. Porém, um estudo sobre avaliação da qualidade de vida de pacientes cardiopatas internados, demonstrou uma maior prevalência de pacientes do sexo masculino, com média de idade de $62,14 \operatorname{anos}^{(8)}$.

Quanto ao nível de escolaridade, os resultados do atual estudo foram semelhantes aos encontrados por outra pesquisa ${ }^{(8)}$, na qual $75 \%$ dos pacientes apresentaram como nível de escolaridade o fundamental incompleto, enquanto que 7,14\% concluíram o ensino médio ${ }^{(8)}$. A renda mensal é um importante fator associado ao tratamento, e os resultados desse mesmo estudo ${ }^{(8)}$ mostraram que $55,32 \%$ dos pacientes tinham como renda mensal apenas 1 salário mínimo ou menos.

Outros estudos revelaram que a maioria dos pacientes analisados tinha uma média de 2-3 salários mínimos como renda mensal ${ }^{(8,19)}$. A baixa escolaridade e renda são fatores de risco para re-hospitalizações. Quanto maior o nível de instrução do paciente, melhor será a sua capacidade de compreensão e maior facilidade terá na tomada de decisões para a promoção, recuperação e proteção da sua saúde, com maior adesão à terapêutica pós-alta. Além disso, a renda interfere na capacidade do acesso ao serviço de saúde, compra de medicamentos e alimentação ${ }^{(19,20)}$.

Uma pesquisa realizada com 170 indivíduos, que avaliou a qualidade de vida relacionada à saúde em idosos com IC, evidenciou que $83 \%$ dos indivíduos eram profissionalmente inativos, o que incluía desempregados, aposentados ou dependentes do auxílio doença ${ }^{(21)}$. Já no presente estudo, quando somados os percentuais de indivíduos inativos (que não trabalham e aposentados), obteve-se um resultado de $97,87 \%(n=46)$ dos pacientes incluídos nesse grupo, o que pode ser justificado pela limitação que a doença traz, associada à dispneia e aos demais sintomas relacionados.

Ainda na presente investigação, os pacientes (40,43\%) relataram um tempo de diagnóstico de IC de aproximadamente 60 meses (5 anos), tendo-se como fator etiológico predominante a miocardiopatia idiopática e a miocardiopatia isquêmica, ambas com 38,3\% $(\mathrm{n}=18)$. Resultado semelhante ao encontrado em outro estudo ${ }^{(21)}$, no qual o tempo médio de diagnóstico de IC foi equivalente a 65,9 meses $( \pm 42,4$; mediana $=60,0)$ e o fator etiológico mais frequente foi a doença isquêmica do coração, apresentado por $46,5 \%$ dos pacientes, seguido pela doença hipertensiva $(32,4 \%)^{(21)}$.

A classificação funcional estratifica o grau de limitação imposto pela doença para atividades cotidianas do indivíduo ${ }^{(2)}$. No estudo em questão, a maioria dos pacientes encontrava-se na classe funcional II e III $(95,74 \%$, n=45), o que justifica também a limitação e inatividade apresentada pela quase totalidade dos pacientes avaliados, uma vez que, na classe funcional II, os sintomas são desencadeados por atividades cotidianas, e na classe III elas estão ainda mais comprometidas, pois os sintomas são desencadeados por atividades menos intensas, ou por pequenos esforços ${ }^{(2)}$.

As principais comorbidades e fatores de risco para doenças cardiovasculares dos pacientes do atual estudo foram semelhantes a outros achados, com destaque para a HAS presente em 57,45\% dos indivíduos avaliados. Esse resultado está em concordância com o que tem sido demonstrado na literatura ${ }^{(21)}$, que aponta como as comorbidades mais prevalentes em pacientes com IC a HAS $(77,6 \%)$, a doença arterial $(44,1 \%)$, a dislipidemia $(35,9 \%)$ e a diabetes mellitus tipo II $(34,7 \%)$. Outro estudo também apresentou a HAS como a comorbidade mais prevalente em $40,4 \%$ dos indivíduos, seguida pela diabetes mellitus, com $17 \%$ (22).

Os fatores de risco cardiovascular podem ser divididos em fatores de risco modificáveis e não modificáveis. Os não modificáveis incluem o sexo, a idade e a hereditariedade, já os modificáveis incluem o tabagismo, o etilismo, o sedentarismo, o estresse, a obesidade, a hipertensão arterial, o diabetes mellitus e as dislipidemias. Os fatores de risco modificáveis são adquiridos com o passar do tempo e estão relacionados com os hábitos de vida dos pacientes. Observou-se que 40,43\% dos pacientes avaliados na atual pesquisa eram ex-tabagistas e 44,47\% eram ex-etilistas, o que demonstra a adoção de hábitos e estilo de vida não saudáveis como fatores que aumentam a probabilidade da ocorrência de doenças cardiovasculares e de outras doenças crônicas não transmissíveis.

O tabagismo foi um dos fatores de risco citado por 31,9\% pelos participantes de outro estudo ${ }^{(21)}$. A história familiar também foi um dos fatores de risco bem presente no atual estudo, com 48,97\% de prevalência de doenças cardíacas na família dos pacientes. A HAS, além de ser uma comorbidade e fator de risco cardiovascular, também é considerada como um importante fator de descompensação da doença, presente em $23,86 \%$ dos pacientes avaliados. No entanto, o principal fator de risco associado à descompensação foi o esforço físico, como relatado por $64,91 \%$ dos pacientes.

Sabe-se que os sintomas clínicos da IC, como dispneia, fadiga, edema, associada à perda energética e perda de massa muscular, podem trazer grandes desconfortos, prejuízos da mobilidade e da capacidade funcional, e interferir na qualidade de vida dos indivíduos ${ }^{(6,10)}$.

Ao analisar o estado nutricional dos participantes da atual pesquisa foi possível verificar que os pacientes apresentaram com maior frequência a classificação nutricional de eutrofia, segundo as variáveis de IMC para idoso, adequação de CB, CMB, e DCT. No entanto, a maior parte dos pacientes adultos (47,22\%) apresentou um diagnóstico nutricional de sobrepeso. Resultados semelhantes foram encontrados em outros estudos, nos quais o primeiro evidenciou $45,6 \%$ dos pacientes tinham o peso adequado e $20,0 \%$ risco de obesidade (sobrepeso) ${ }^{(23)}$, enquanto o segundo mostrou que $25,5 \%$ tinham o peso adequado, $23,4 \%$ estavam com risco de obesidade e $10,6 \%$ eram obesos ${ }^{(20)}$. 
A evolução clínica dos pacientes com IC leva a uma série de alterações fisiológicas, que traz um desequilíbrio entre processos anabólicos e catabólicos, fatores importantes na gênese da caquexia e estão relacionados à proteólise da musculatura esquelética ${ }^{(4,24)}$. Porém, a presença de desnutrição foi baixa em relação aos percentuais de eutrofia e sobrepeso/obesidade nos pacientes analisados na atual pesquisa. Vale lembrar que, embora os pacientes estejam classificados como eutróficos e/ou em risco de obesidade e obesidade, ainda são considerados pacientes em risco nutricional. Pode-se afirmar que os pacientes com IMC elevado podem ter alterações cardíacas funcionais e estruturais mesmo na ausência de doença cardíaca clínica, devido à disfunção sistólica e diastólica subclínica ${ }^{(6)}$.

A avaliação do consumo alimentar através da utilização do QFA mostrou uma alta taxa de inadequação do consumo do grupo leite, queijo e iogurte; grupo feijões e oleaginosas e grupo frutas, verduras e legumes, com respectivamente $87,75 \%$, $87,36 \%$ e $54,42 \%$ na presente pesquisa.

De acordo com a pirâmide alimentar brasileira, para uma alimentação saudável, recomenda-se o consumo de três porções diárias de leite ou derivados, dando preferência ao desnatado para adultos e ao integral para crianças ${ }^{(13)}$. Evidências mostram que a ingestão de leites e derivados com baixo teor de gordura atua na redução da pressão arterial, pois o leite contém vários componentes, como cálcio, potássio e peptídeos bioativos que contribuem no controle e diminuição da pressão $0^{(25,26)}$.

A pirâmide alimentar recomenda o consumo de, pelo menos, 1 porção de feijões e oleaginosas por dia, e o consumo diário de feijão com arroz, na proporção de 1 para 2 partes. Essa combinação típica brasileira, feijão com arroz, é saudável e completa em proteínas. Os alimentos vegetais mais ricos em proteínas são as leguminosas. Quando cozidos, contêm 6\% a 11\% de proteína, contêm ainda carboidratos complexos (amido) e são ricos em fibra alimentar, vitaminas do complexo B, ferro, cálcio e outros minerais, bem como em compostos bioativos. Contêm pequenas quantidades de gordura, sendo quase toda do tipo insaturada. Embora a participação relativa de feijões na alimentação brasileira $(5,68 \%)$ ainda esteja dentro da faixa recomendada de consumo, há uma tendência de queda preocupante, necessitando ser revertida em curto tempo. Essa afirmação pôde ser confirmada de acordo com os resultados do estudo em que $67,76 \%$ nunca consumiam alimentos desses grupos ${ }^{(13)}$.

Tiveram ainda consumo inadequado o grupo dos óleos e gorduras $(78,23 \%, \mathrm{n}=37)$ e o grupo dos açúcares e doces $(81,97 \%$, n=39) na presente pesquisa. Esses refletem um aspecto positivo em relação à baixa ingestão, uma vez que o excesso está associado ao acometimento de doenças crônicas não transmissíveis (DCNT). Em relação à adequada ingestão de consumo, destaca-se a ingestão do grupo do arroz, pão, massa, batata e mandioca, com 81,97\% ( $\mathrm{n}=39)$, e o grupo das carnes e ovos $66,12 \%(\mathrm{n}=30)$, considerando carne vermelha, frango, peixe e ovos. Deste último, a menor frequência ficou para a carne vermelha. Resultados semelhantes foram vistos em outro estudo, no qual os grupos de alimentos mais consumidos foram o grupo das carnes e miúdos, com $57,4 \%$. Porém, destaca-se o alto consumo de embutidos, pois $38,1 \%$ dos pacientes consumiam alimentos desse grupo por pelo menos 2-4 vezes por semana, e o das frutas com $34,8 \%{ }^{(27)}$. O grupo das frutas, apesar de ter sido mais consumida nesse estudo ${ }^{(27)}$, referia-se ao consumo semanal e não diário, o que torna a ingestão inadequada segundo as recomendações da pirâmide(13).

De acordo com a pirâmide, orienta-se o consumo de três porções diárias do grupo das frutas e do grupo dos legumes e verduras, pois esses alimentos contribuem para a proteção à saúde e diminuição do risco de aparecimento de doenças crônicas não transmissíveis, como a HAS, o DM e a obesidade ${ }^{(24)}$.

Dietas que enfatizam o consumo de frutas e vegetais favorecem a melhora de diversos fatores de risco, incluindo pressão arterial, perfil lipídico, resistência à insulina, marcadores inflamatórios, função endotelial e controle de peso. O consumo de frutas e vegetais parece reduzir o aparecimento de doenças crônicas não transmissíveis, mais especificamente a doença coronariana, devido a existência de nutrientes como potássio, folato, vitaminas, fibras e outros compostos fenólicos ${ }^{(28)}$.

Os padrões alimentares adotados nas últimas décadas podem ser prejudiciais à saúde de diversas formas. O consumo excessivo de sal/sódio aumenta o risco de hipertensão e eventos cardiovasculares, e o alto consumo de carne vermelha e carne processada, assim como outras gorduras de origem animal, fontes de ácidos graxos saturados e ácidos graxos trans, estão relacionadas às doenças cardiovasculares e à diabetes, pois aumentam a concentração de lipídeos e de lipoproteínas plasmáticas. Já a ingestão regular de frutas e legumes diminui o risco de doenças cardíacas e câncer ${ }^{(29-31)}$.

Os tipos de carboidratos que compõem a alimentação também merecem atenção. Uma alimentação baseada em alimentos não refinados e que alcance apenas o limite inferior recomendável (45\%) é mais saudável que a constituída no limite superior $(65 \%)$ de energia oriunda desse grupo de alimentos. Para o consumo de carboidratos totais (complexos + açúcares livres ou simples), as orientações são de 55\% a 75\% do valor energético total (VET) da alimentação diária. Destes, mais da metade da energia fornecida deverá ter origem em alimentos ricos em carboidratos complexos (grãos, tubérculos e raízes), ou seja, $45 \%$ a $65 \%$ do VET. Uma alimentação que atenda a essa recomendação traz muitos benefícios, principalmente quando se utilizam carboidratos em sua forma integral ${ }^{(13)}$.

O planejamento alimentar deve levar em consideração o estado nutricional, as condições clínicas e as comorbidades associadas para que as medidas dietoterápicas tragam os benefícios esperados ${ }^{(6)}$. $\mathrm{O}$ padrão alimentar de pacientes cardiopatas deve ser resgatado por meio do incentivo à alimentação saudável, juntamente com orientação sobre a seleção dos alimentos, o modo de preparo, a quantidade e as possíveis substituições alimentares, sempre em sintonia com a mudança do estilo de vida ${ }^{(27)}$. 
Em relação à qualidade de vida, verificou-se na presente pesquisa que todos os domínios apresentaram como resultado uma qualidade de vida de regular a boa, e que os maiores coeficientes de variação foram sobre a autoavaliação da qualidade de vida, domínio físico e domínio psicológico. O que significa dizer que, devido à grande variabilidade de dados desses domínios, a média da avaliação da qualidade de vida não se torna a mais alta. Os resultados obtidos da análise dos escores por facetas mostraram aspectos positivos e outros negativos em relação à qualidade de vida. Entre os negativos destaca-se que $41,49 \%$ dos indivíduos sentem algum tipo de dor ou desconforto relacionado ao cansaço e falta de energia. Isto pode ser reafirmado quando se analisa o escore de energia e fadiga relatadas pelos pacientes, o que interfere diretamente na execução das atividades básicas diárias.

Um estudo sobre a qualidade de vida de idosos, ao comparar os quatro domínios e QV geral entre os participantes, evidenciou que as maiores pontuações entre os idosos da comunidade eram nos domínios 1 (físico), 2 (psicológico) e 3 (relações sociais), mas apenas o segundo e o terceiro domínio com diferença estatisticamente significativa ${ }^{(32)}$.

Outro estudo, também sobre a qualidade de vida em idosos, mostrou que as variáveis estudadas de forma independente explicaram uma variância no domínio físico; $9,2 \%$ da variância no domínio psicológico e 4,8\% da variância no domínio social, respectivamente. Nesses três domínios, a presença de doenças mostrou-se inversamente associada à qualidade de vida, ou seja, os idosos sem quaisquer doenças apresentaram qualidade de vida significantemente melhor em relação aos que tinham pelo menos uma doença e as demais variáveis não influenciaram significantemente a qualidade de vida dos idosos ${ }^{(33)}$.

Outro ponto negativo observado se refere à dependência que os pacientes da presente pesquisa tinham em relação ao uso de medicações e/ou necessidade de um acompanhante durante as consultas clínicas. Em relação à qualidade de vida, foi a pouca oportunidade de lazer, relatada apenas por 39,36\% dos pacientes, e os que não realizavam justificaram essa não prática por motivos de cansaço e/ou custo financeiro.

Apesar da pouca significância entre as variáveis correlacionadas no presente estudo, é importante enfatizar que a qualidade de vida deve ser avaliada como um todo e não somente por domínios isolados. Mesmo com alguns domínios mais comprometidos, os pacientes do atual estudo apresentaram, de um modo geral, uma qualidade de vida de regular a boa, uma vez que o domínio psicológico e as relações sociais alcançaram valores mais próximos da pontuação máxima, que classifica a qualidade de vida como muito boa. O impacto da doença na qualidade de vida pode ser influenciado por múltiplos fatores, como gravidade da doença, comorbidades, etnia, tempo de diagnóstico, apoio familiar e/ou apoio psicológico ${ }^{(34)}$.

Um estudo evidenciou que o maior impacto na qualidade de vida dos pacientes relacionava-se com a dimensão física, ou seja, a percepção das dificuldades em realizar alguma tarefa, fazer longas caminhadas e a presença de falta de ar durante a execução de tais tarefas. Inegavelmente as principais limitações do paciente com IC são realmente físicas, pois atuam com grande impacto sobre a vida laboral e grave impacto socioeconômico, logicamente, com a progressão da doença ${ }^{(35)}$.

O envelhecimento ativo e saudável está relacionado com a promoção da autonomia e, a partir disso, com atuar na prevenção do isolamento social e da solidão das pessoas, pois a qualidade de vida e bem-estar estão diretamente ligadas ao convívio, social, familiar e ao sentir-se útil( ${ }^{(36)}$.

Dentre os aspectos positivos do presente estudo, os que mais se destacaram foram o apoio social, assim como as relações pessoais, o ambiente em que moravam e o quesito autoestima. Dessa forma, os pacientes estavam "satisfeitos" ou "muito satisfeitos" com suas relações pessoais e sociais, como o apoio recebido dos amigos, famílias e com o ambiente em que vivem. Fatores que impactam de forma importante na autoestima do paciente. Além disso, mesmo tendo sido referidas poucas atividades de lazer, a boa relação dos pacientes com seus familiares e amigos foi um fator positivo na autoestima dos participantes desta pesquisa.

A participação social no processo de envelhecimento reforça os laços sociais através das relações estabelecidas com os diversos subsistemas institucionais, como a família, a vizinhança e o grupo de pares, destacam-se como um importante fator para a felicidade dos idosos, e estão associadas a melhores níveis de bem-estar, satisfação, saúde e qualidade de vida ${ }^{(37,38)}$.

Os resultados obtidos no presente estudo mostraram uma correlação linear direta entre DCT e domínio psicológico e inversamente proporcional entre estatura e domínio qualidade de vida, respectivamente. No entanto, essas relações não se apresentaram forte. Entretanto, apresentou como limitação o tamanho da amostra, em virtude do absenteísmo às consultas agendadas e à rejeição de alguns quanto à participação na pesquisa.

\section{CONCLUSÃO}

Independentemente das variáveis demográficas e clínicas, os pacientes adultos e idosos da atual investigação apresentaram perfil nutricional dentro dos parâmetros normais (de acordo com IMC, CB e CMB) e/ou sobrepeso para DCT em idosos, e qualidade de vida de regular a boa. Além disso, o estado nutricional e qualidade de vida podem sofrer variações entre os indivíduos e no mesmo indivíduo, dependendo da fase da vida que esteja e do apoio social/familiar que recebem. Portanto, esses fatores atuam como determinantes positivos na qualidade de vida mesmo na presença da doença cardíaca. 


\section{REFERÊNCIAS}

1. Sociedade Brasileira de Cardiologia. II Diretriz Brasileira de Transplante Cardíaco. Arq Bras Cardiol. 2010;94(1):e16-e76.

2. Sociedade Brasileira de Cardiologia. Atualização da Diretriz Brasileira de Insuficiência Cardíaca Crônica.. Arq Bras Cardiol. 2012;98(1 Supl 1):1-33.

3. Go AS, Mozaffarian D, Roger VL, Benjamin EJ, Berry JD, Blaha MJ, American Heart Association Statistics Committee and Stroke Statistics Subcommittee, et al. Heart disease and stroke statistics-2014 update: a report from the American Heart Association. Circulation. 2014;129(3):e28e292.

4. Holst, M, Strömberg A, Lindholm M, Willenheimer R. Description of self-repoited fluid intake and its effects on body weight, symptoms, quality of life and physical capacity in patients with stable chronic heart failure. J Clin Nurs. 2008;17(17):2318-26.

5. Duarte MB, Silva CV, Subutzki PS, Sousa EN, Azzolin KO, et al. Há correlação entre a função ventricular e o estado nutricional? Instituto de cardiologia/ ICFUC, Porto alegre, RS. Arq Bras Cardiol. 2014;103(2 Supl 3):1-34.

6. Cuppari L. Guia de Nutrição: clinica no adulto. $3^{\mathrm{a}}$ ed. Baueri: Manole; 2014. (Série guias de medicina ambulatorial e hospitalar).

7. Oliveira TCT, Correia DMS, Cavalcanti ACD. O impacto da insuficiência cardíaca no cotidiano: percepção do paciente em acompanhamento ambulatorial. Rev Enferm UFPE on line. 2013;7(6):4497-7.

8. Christmann M, Costa CC, Moussalle LD. Avaliação da qualidade de vida de pacientes cardiopatas internados em um hospital público. Rev AMRIGS. 2011;55(3):239-43.

9. World Health Organization Obesity. Preventing and managing the global epidemic: report of a WHO Consultation. Geneva, WHO; 1998. (Technical Report Series, 894).

10. Lipschitz DA. Screening for nutritional status in the elderly. Prim Care. 1994;21(1):55-67.

11. Blackburn GL, Thorton PA. Nutritional assessment of the hospitalized patients. Med Clin North Am. 1979;63(5):1103-15.

12. Pedraza DF, Menezes TN. Questionários de Frequência de Consumo Alimentar desenvolvidos e validados para população do Brasil: revisão da literatura. Ciênc Saúde Colet. 2015;20(9):2697-720.

13. Philippi ST. Redesenho da Pirâmide Alimentar Brasileira para uma alimentação saudável, 2013 [acesso em 2017 Fev 20]. Disponível em: http://54.232.216.243/sites/libwww/udc/uploads/uploadsMateriais/26052017110402redesenho\%20 da\%20pir\%C3\%A2mide\%20alimentar.pdf

14. Kluthcovsky ACGC, Kluthcovsky FA. O WHOQOL-bref, um instrumento para avaliar qualidade de vida: uma revisão sistemática. Rev Psiquiatr Rio Gd Sul. 2009;31(3 suppl):1-12.

15. Fleck MPA. O instrumento de avaliação de qualidade de vida da Organização Mundial da Saúde (WHOQOL-100): características e perspectivas. Ciênc Saúde Colet. 2000;5(1):33-8.

16. Braga MCP, Casella MA, Campos MLN, Paiva SP. Qualidade de vida medida pelo Whoqol-Bref: Estudo com idosos residentes em Juiz de Fora/MG. Rev APS. 2011;14(1):93-100.

17. Gomes JRAA, Hamann EM, Gutierrez MMU. Aplicação do WHOQOL-BREF em segmento da comunidade como subsídio para ações de promoção da saúde. Rev Bras Epidemiol. 2014;17(2):495-516.

18. Pedroso B, Pilatti LA, Gutierrez GL, Santos CB, Picinin CT. Validação da sintaxe unificada para o cálculo dos escores dos Instrumentos WHOQOL. Conexões - Rev Facul Educ Físic UNICAMP. 2011;9(1):130-56.

19. Linn AC, Azzolin K, Souza EN. Associação entre autocuidado e reinternação hospitalar de pacientes com insuficiência cardíaca. Rev Bras Enferm. 2016;69(3):500-6.

20. Almeida GAS, Teixeira JBA, Barichello E, Barbosa MH. Perfil de saúde de pacientes acometidos por insuficiência cardíaca. Esc Anna Nery. 2013;17(2):328-35.

21. Saccomann ICR, Cintra FA, Gallani MCBJ. Qualidade de vida relacionada à Saúde em Idosos com Insuficiência cardíaca: avaliação com instrumento específico. Acta Paul Enferm. 2011;24(2):179-84.

22. Silva SO. Avaliação da qualidade de vida e do desempenho físico em pacientes clínicos e cirúrgicos com insuficiência cardíaca [dissertação]. Vila Real; 2013. 
23. Lourenço BH, Vieira LP, Macedo A, Nakasato M, Marucci MFN, et al. Estado nutricional e adequação da ingestão de energia e nutrientes em pacientes com insuficiência cardíaca. São Paulo: Hospital das Clínicas da Faculdade de Medicina da Universidade de São Paulo; 2008.

24. Schulze PC. Protein catabolism and impairment of skeletal muscle insulin signalling in heart failure. Clin Sci (Lond). 2010;19(11):465-6.

25. Park KM, Cifelli CJ. Dairy and blood pressure: a fresh look at the evidence. Nutr Rev. 2013;71(3):149-57.

26. Fekete AA, Givens DI, Lovegrove JA. The impact of milk proteins and peptides on blood pressure and vascular function: a review of evidence from human intervention studies. Nutr Res Rev. 2013;26(2):177-90.

27. Borges CF, Busnello FM, Pellanda LC. Identificação de fatores de risco cardiovascular em pais/cuidadores de crianças cardiopatas. Arq Bras Cardiol. 2012;99(4):936-43.

28. Buil-Cosiales P, Martinez-Gonzalez MA, Ruiz-Canela M, Díez-Espino J, García-Arellano A. consumption of fruit or fiberfruit decreases the risk of cardiovascular disease in a mediterranean young cohort. Nutrients. 2017;9(3):295.

29. Ministério da Saúde (BR), Secretaria de Atenção à Saúde. Departamento de Atenção Básica. Guia alimentar para a população brasileira. $2^{\mathrm{a}}$ ed. Brasília: Ministério da Saúde; 2014.

30. Faludi AA, Izar MCO, Saraiva JFK, Chacra APM, Bianco HT, Afiune A Neto, et al. Atualização da Diretriz Brasileira de Dislipidemias e Prevenção da Aterosclerose. Arq Bras Cardiol. 2017;109(2 Suppl 1):1-76.

31. Duncan BB, Chor D, Aquino EML, Bensenor IM, Mill JG, et al. Doenças crônicas não transmissíveis no Brasil: prioridade para enfrentamento e investigação. Rev Saúde Pública. 2012;46(Supl 1):126-34.

32. Vitorino LM, Paskulin LMG, Vianna LAC. Qualidade de vida de idosos da comunidade e de instituições de longa permanência: estudo comparativo. Rev.Latinoam Enferm. 2013;21(Spec):1-9.

33. Dawalibi NW, Goulart RMM, Prearo LC. Fatores relacionados à qualidade de vida de idosos em programas para a terceira idade. Ciênc Saúde Colet. 2014;19(8):3505-12.

34. Mussi CM, Souza EN, Ruschel KB, Camargo CP, Lopes ANM, et al. Qualidade de vida em pacientes com insuficiência cardíaca acompanhados no domicílio. Rev HCPA. 2012;32 (Suppl):40.

35. Calles ACN, Moreira ET, Lira JLF, Constant MHL, Farias AR, Costa FLB. Correlação entre a qualidade de vida e a classificação funcional em pacientes com insuficiência cardíaca congestiva em internamento hospitalar. Cadernos de Graduação - Ciências Biológicas e da Saúde Fits. 2013;1(2):111-20.

36. Carneiro R, Chau F, Soares C, Fialho JAS, Sacadura MJ. O envelhecimento da população: dependência, ativação e qualidade: relatório final. Lisboa: Universidade Católica Portuguesa; 2012.

37. Veloso AST. Envelhecimento, saúde e satisfação efeitos do envelhecimento ativo na qualidade de vida [dissertação]. Coimbra: Universidade de Coimbra; 2015.

38. Cabral MV, Ferreira PM, Silva PA, Jerónimo P, Marques T. Processos de envelhecimento em Portugal: uso do tempo, redes sociais e condições de vida. Lisboa: Fundação Francisco Manuel dos Santos; 2013.

\section{Endereço para correspondência:}

Luana Najara Ferreira Chaves

Hospital de Messejana Dr. Carlos Alberto Studart Gomes

Avenida Frei Cirilo, 3480

Bairro: Messejana

CEP: 60.846-190 - Fortaleza - CE - Brasil

E-mail: luanachaves35@yahoo.com.br 